Vol. 23, No. 1 (2019), 107-118 doi: 10.5937/MatMor1901107A

\title{
Upper and lower solutions method for Caputo-Hadamard fractional differential inclusions
}

\author{
SAÏD ABBAS, MOUFFAK BENCHOHRA, \\ SAmira HAMAni, JohnNy HEndERson
}

\begin{abstract}
In this paper, we use some background concerning multivalued functions and set-valued analysis, the fixed point theorem of Bohnenblust-Karlin and the method of upper and lower solutions to investigate the existence of solutions for a class of boundary value problem of functional differential inclusions involving the Caputo-Hadamard fractional derivative.
\end{abstract}

\section{INTRODUCTION}

There are numerous applications of fractional calculus and fractional differential equations in various fields of science and engineering; see [4, 7, 8, 17, 20, 23, 24, 25]. Many researchers studied different classes of differential equations involving the Riemann-Liouville, Caputo and Hadamard derivatives; see $[3,6,10,11,12,13,21]$.

In $[1,2,5,9,15,22]$, the authors use the method of upper and lower solutions to study the existence of solutions for ordinary and fractional differential equations and inclusions. In this paper we give some existence results for the following Caputo-Hadamard fractional differential inclusion,

$$
{ }^{H c} D^{r} y(t) \in F(t, y(t)) ; \quad \text { for a.e. } t \in J=[1, T],
$$

with the boundary condition

$$
L(y(1), y(T))=0,
$$

where $T>1,{ }^{H c} D^{r}$ is the Caputo-Hadamard fractional derivative of order $0<r \leq 1, F: J \times \mathbb{R} \rightarrow \mathcal{P}(\mathbb{R})$ is a multivalued map, $\mathcal{P}(\mathbb{R})$ is the family of all nonempty subsets of $\mathbb{R}$, and $L: \mathbb{R}^{2} \rightarrow \mathbb{R}$ is a given continuous function.

2000 Mathematics Subject Classification. The form is: Primary: 26A33; Secondary: 34A60, 34D10.

Key words and phrases. Fractional differential inclusion; Caputo-Hadamard fractional derivative; fixed point; boundary condition; upper solution; lower solution.

Full paper. Received 9 May 2018, revised 11 June 2019, accepted 14 June 2019, available online 17 June 2019. 
This paper initiates the application the method of upper and lower solutions for fractional differential inclusions involving the Caputo-Hadamard fractional derivative.

\section{Preliminaries}

Let $\left(C(J, \mathbb{R}),\|\cdot\|_{\infty}\right)$ be the Banach space of continuous functions $y$ from $J$ to $\mathbb{R}$ with the usuel uniform norm

$$
\|y\|_{\infty}=\sup _{t \in J}|y(t)|
$$

By $L^{1}(J, \mathbb{R})$ we denote the Banach space of all Lebesgue integrable functions $y: J \rightarrow \mathbb{R}$ with the norm

$$
\|y\|_{L^{1}}=\int_{1}^{T}|y(t)| d t .
$$

Denote by $A C(J, \mathbb{R})$ the space of absolutely continuous functions from $J$ into $\mathbb{R}$.

For a given Banach space $(X,\|\cdot\|)$, we define the following subsets of $\mathcal{P}(X)$ :

$$
\begin{aligned}
& P_{c l}(X)=\{Y \in \mathcal{P}(X): Y \text { is closed }\} \\
& P_{b}(X)=\{Y \in \mathcal{P}(X): Y \text { is bounded }\} \\
& P_{c p}(X)=\{Y \in \mathcal{P}(X): Y \text { is compact }\} \\
& P_{c v}(X)=\{Y \in \mathcal{P}(X): Y \text { is convex }\} \\
& P_{c p, c v}(X)=P_{c p}(X) \cap P_{c v}(X)
\end{aligned}
$$

Definition 2.1. A multivalued map $G: X \rightarrow \mathcal{P}(X)$ is said to be convex (closed) valued if $G(x)$ is convex (closed) for all $x \in X$. A multivalued map $G$ is bounded on bounded sets if $G(B)=\cup_{x \in B} G(x)$ is bounded in $X$ for all $B \in P_{b}(X)$ (i.e. $\sup _{x \in B}\{\sup \{|y|: y \in G(x)\}$ exists).

Definition 2.2. A multivalued map $G: X \rightarrow \mathcal{P}(X)$ is called upper semicontinuous (u.s.c.) on $X$ if for each $x_{0} \in X$, the set $G\left(x_{0}\right)$ is a nonempty closed subset of $X$, and for each open set $N$ of $X$ containing $G\left(x_{0}\right)$, there exists an open neighborhood $N_{0}$ of $x_{0}$ such that $G\left(N_{0}\right) \subset N$. G is said to be completely continuous if $G(B)$ is relatively compact for every $B \in P_{b}(X)$.

Definition 2.3. Let $G: X \rightarrow \mathcal{P}(X)$ be completely continuous with nonempty compact values. Then $G$ is u.s.c. if and only if $G$ has a closed graph (i.e. $x_{n} \rightarrow x_{*}, y_{n} \rightarrow y_{*}, y_{n} \in G\left(x_{n}\right)$ imply $\left.y_{*} \in G\left(x_{*}\right)\right)$. $G$ has a fixed point if there is $x \in X$ such that $x \in G(x)$.

We denote by Fix $G$ the fixed point set of the multivalued operator $G$. 
Definition 2.4. A multivalued map $G: J \rightarrow P_{c l}(\mathbb{R})$ is said to be measurable if for every $y \in \mathbb{R}$, the function:

$$
t \rightarrow d(y, G(t))=\inf \{|y-z|: z \in G(t)\}
$$

is measurable.

Lemma 2.1. [18] Let $G$ be a completely continuous multivalued map with nonempty compact values, then $G$ is u.s.c. if and only if $G$ has a closed graph.

Definition 2.5. A multivalued map $F: J \times \mathbb{R} \rightarrow \mathcal{P}(\mathbb{R})$ is said to be Carathéodory if:

(1) $t \rightarrow F(t, u)$ is measurable for each $u \in \mathbb{R}$;

(2) $u \rightarrow F(t, u)$ is upper semicontinuous for almost all $t \in J$.

$F$ is said to be $L^{1}$-Carathéodory if (1), (2) and the following condition holds:

(3) For each $q>0$, there exists $\varphi_{q} \in L^{1}\left(J, \mathbb{R}^{+}\right)$such that $\|F(t, u)\|_{\mathcal{P}}=\sup \{|v|: v \in F(t, u)\} \leq \varphi_{q}$ for all $|u| \leq q$ and for a.e. $t \in J$.

For each $y \in C(J, \mathbb{R})$, define the set of selections of $F$ by

$$
S_{F \circ y}=\left\{v \in L^{1}([1, T], \mathbb{R}): v(t) \in F(t, y(t)) \text { a.e. } t \in[1, T]\right\} .
$$

Let $(X, d)$ be a metric space induced from the normed space $(X,|\cdot|)$. the function $H_{d}: \mathcal{P}(X) \times \mathcal{P}(X) \rightarrow \mathbb{R}_{+} \cup\{\infty\}$ given by:

$$
H_{d}(A, B)=\max \left\{\sup _{a \in A} d(a, B), \sup _{b \in B} d(A, b)\right\}
$$

is known as the Hausdorff-Pompeiu metric. For more details on multivalued maps see the books of $\mathrm{Hu}$ and Papageorgiou [18].

In the sequel, we need the following fixed point theorem:

Theorem 2.1. (Bohnenblust-Karlin)[16] Let $X$ be a Banach space and $K \in$ $\mathcal{P}_{c l, c v}(X)$, and suppose that the operator $G: K \rightarrow \mathcal{P}_{c l, c v}(K)$ is upper semicontinuous and the set $G(K)$ is relatively compact in $X$. Then $G$ has a fixed point in $K$.

Let us recall some definitions and properties of Hadamard fractional integration and differentiation. Let $\delta=t \frac{d}{d t}$, and set

$$
A C_{\delta}^{n}(J, \mathbb{R})=\left\{y: J \rightarrow \mathbb{R}: \delta^{n-1} y(t) \in A C(J, \mathbb{R})\right\} .
$$

Definition 2.6. [20] The Hadamard fractional integral of order $r>0$ for a function $h \in L^{1}([1,+\infty), \mathbb{R})$ is defined as

$$
{ }^{H} I_{1}^{r} h(t)=\frac{1}{\Gamma(r)} \int_{1}^{t}\left(\log \frac{t}{s}\right)^{r-1} \frac{h(s)}{s} d s,
$$

provided the integral exists. 
Example 2.1. Let $q>0$. Then

$$
{ }^{H} I_{1}^{q} \ln t=\frac{1}{\Gamma(2+q)}(\ln t)^{1+q} ; \text { for a.e. } t \in[1,+\infty) .
$$

Definition 2.7. [20] The Hadamard fractional derivative of order $r>0$ applied to the function $h \in A C_{\delta}^{n}([1,+\infty), \mathbb{R})$ is defined as

$$
\left({ }^{H} D_{1}^{q} h\right)(t)=\delta^{n}\left({ }^{H} I_{1}^{n-r} h\right)(t),
$$

where $n-1<r<n, n=[r]+1$, and $[r]$ is the integer part of $r$.

Definition 2.8. [19] For a given function $h \in A C_{\delta}^{n}([a, b], \mathbb{R})$, such that $0<a<b$, the Caputo-Hadamard fractional derivative of order $r>0$ is defined as follows:

$$
{ }^{H c} D^{r} y(t)={ }^{H} D_{1}^{r}\left[y(s)-\sum_{k=0}^{n-1} \frac{\delta^{k} y(a)}{k !}\left(\log \frac{s}{a}\right)^{k}\right](t),
$$

where $\operatorname{Re}(\alpha) \geq 0$ and $n=[\operatorname{Re}(\alpha)]+1$.

Lemma 2.2. [19] Let $y \in A C_{\delta}^{n}([a, b], \mathbb{R})$ or $C_{\delta}^{n}([a, b], \mathbb{R})$ and $\alpha \in \mathbb{C}$. Then

$$
{ }^{H} I_{1}^{r}\left({ }^{H c} D^{r} y\right)(t)=y(t)-\sum_{k=0}^{n-1} \frac{\delta^{k} y(a)}{k !}\left(\log \frac{t}{a}\right)^{k} .
$$

\section{Main Results}

Definition 3.1. A function $y \in A C(J, \mathbb{R})$ is said to be a solution of $(1)-(2)$ if there exists a function $v \in S_{F o u}$ such that ${ }^{H c} D^{r} y(t)=v(t)$ a.e. $J$ and the boundary condition $L(y(1), y(T))=0$ is satisfied.

Definition 3.2. Afunction $w \in A C(J, \mathbb{R})$ is said to be an upper solution of $(1)-(2)$ if $L(w(1), w(T)) \geq 0$, and there exists a function $v_{1} \in S_{F \circ w}$ such that ${ }^{H c} D^{r} w(t) \geq v_{1}(t)$ a.e. $J$.

Similarly, A function $u \in A C(J, \mathbb{R})$ is said to be a lower solution of (1) (2) if $L(u(1), u(T)) \leq 0$, and there exists a function $v_{2} \in S_{F \circ u}$ such that ${ }^{H c} D^{r} u(t) \leq v_{2}(t)$ a.e. $J$.

Theorem 3.1. Assume the following hypotheses hold:

(H1) $F: J \times \mathbb{R} \rightarrow \mathcal{P}_{c p, c}(\mathbb{R})$ is Carathéodory,

(H2) There exist $u, w \in C(J, \mathbb{R})$, lower and upper solutions, respectively, for problem $(1)-(2)$ such that $u \leq w$,

(H3) The function $L(\cdot, \cdot)$ is continuous on $[u(1), w(1)] \times[u(T), w(T)]$, and nonincreasing with respect to both of its arguments,

(H4) There exists $l \in L^{1}\left(J, \mathbb{R}^{+}\right)$such that

$$
H_{d}(F(t, y), F(t, \bar{y})) \leq l(t)|y-\bar{y}| ; \text { for every } y, \bar{y} \in \mathbb{R},
$$

and

$$
d(0, F(t, 0)) \leq l(t) ; \text { a.e. } t \in J .
$$


Then the problem (1) - (2) has at least one solution y defined on $J$ such that

$$
u \leq y \leq w .
$$

Proof. Consider the following modified problem

$$
\begin{gathered}
{ }^{H c} D^{r} y(t)=F(t, \tau(y(t))) ; \text { for a.e. } t \in J, \\
y(1)=\tau(y(1))-L(\bar{y}(1), \bar{y}(T)),
\end{gathered}
$$

where

and

$$
\tau(y(t))=\max \{u(t), \min \{y(t), w(t)\}\}
$$

$$
\bar{y}(t)=\tau(y(t)) .
$$

A solution to $(3)-(4)$ is a fixed point of the operator $N: C(J, \mathbb{R}) \rightarrow$ $\mathcal{P}(C(J, \mathbb{R}))$ defined by

$$
N(y)=\left\{h \in C(J, \mathbb{R}): h(t)=y(1)+\frac{1}{\Gamma(\alpha)} \int_{1}^{t}\left(\log \frac{t}{s}\right)^{r-1} \nu(s) \frac{d s}{s}\right\},
$$

where

$$
\begin{gathered}
\nu \in\left\{v \in \widetilde{S}_{F \circ \tau(y)}^{1}: v(t) \geq v_{1}(t) \text { on } A_{1} \text { and } v(t) \leq v_{2}(t) \text { on } A_{2}\right\}, \\
S_{F \circ \tau(y)}^{1}=\left\{v \in L^{1}(J, \mathbb{R}): v(t) \in F(t,(\tau y)(t)), \text { a.e. } t \in J\right\}, \\
A_{1}=\{t \in J: y(t)<u(t) \leq w(t)\}, A_{2}=\{t \in J: u(t) \leq w(t)<y(t)\} .
\end{gathered}
$$

Remark 3.1. (1) For each $y \in C(J, \mathbb{R})$, the set $\widetilde{S}_{F \circ \tau(y)}^{1}$ is nonempty. In fact, $(H 1)$ implies that there exists $v_{3} \in S_{F \circ \tau(y)}^{1}$, so we set

$$
v=v_{1} \chi_{A_{1}}+v_{2} \chi_{A_{2}}+v_{3} \chi_{A_{3}} \text {, }
$$

where

$$
A_{3}=\{t \in J: u(t) \leq y(t) \leq w(t)\} .
$$

Then by decomposability, $v \in \widetilde{S}_{F \circ \tau(y)}^{1}$.

(2) By the definition of $\tau$ it is clear that $F(\cdot, \tau y(\cdot))$ is an $L^{1}$ - Carathéodory multi-valued map with compact convex values and there exists $\phi_{1} \in$ $L^{1}\left(J, \mathbb{R}^{+}\right)$such that

$$
\|F(t, \tau y(t))\|_{\mathcal{P}} \leq \phi_{1}(t) \text { for each } y \in \mathbb{R} .
$$

(3) Since $\tau(y(t))=u(t)$ for $t \in A_{1}$, and $\tau(y(t))=w(t)$ for $t \in A_{2}$, then from (H3), the equation (4) implies that

$$
|y(1)| \leq|u(1)|+\mid L\left(u(1), u(T)|\leq| u(1)|+| L(y(1), y(T))|=| u(1) \mid \text { on } A_{1}\right. \text {, }
$$

and

$$
y(1)=w(1)-L\left(w(1), w(T) \leq w(1)-L(y(1), y(T))=w(1) \text { on } A_{2} .\right.
$$

These show that,

$$
|y(1)| \leq \min \{|u(1)|,|w(1)|\} .
$$


Let

$$
R:=\min \{|u(1)|,|w(1)|\}+\frac{\left\|\phi_{1}\right\|_{L_{1}}}{\Gamma(r+1)}(\log T)^{r},
$$

and consider the closed and convex subset of $C(J, \mathbb{R})$,

$$
B=\left\{y \in C(J, \mathbb{R}):\|y\|_{\infty} \leq R\right\} .
$$

We shall show that the operator $N: B \rightarrow \mathcal{P}_{c l, c v}(B)$ satisfies all assumptions of Theorem 2.1. The proof will be given in six steps.

Step 1: $N(y)$ is convex for each $y \in B$.

Let $h_{1}, h_{2}$ belong to $N(y)$, then there exist $\nu_{1}, \nu_{2} \in \widetilde{S}_{F \circ \tau(y)}^{1}$ such that for each $t \in J$, we have, for $i=1,2$,

$$
h_{i}(t)=y(1)+\frac{1}{\Gamma(r)} \int_{1}^{t}\left(\log \frac{t}{s}\right)^{r-1} \nu_{i}(s) \frac{d s}{s} .
$$

Let $0 \leq d \leq 1$. Then, for each $t \in J$, we have

$$
\left(d h_{1}+(1-d) h_{2}\right)(t)=y(1)+\frac{1}{\Gamma(r)} \int_{1}^{t}\left(\log \frac{t}{s}\right)^{r-1}\left[d \nu_{1}(s)+(1-d) \nu_{2}(s)\right] \frac{d s}{s} .
$$

Since $S_{F \circ \tau(y)}$ is convex (because $F$ has convex values), we have

$$
d h_{1}+(1-d) h_{2} \in N(y) .
$$

Step 2: $N$ maps bounded sets into bounded sets in $B$.

For each $h \in N(y)$, there exists $\nu \in \widetilde{S}_{F \circ \tau(y)}^{1}$ such that

$$
h(t)=y(1)+\frac{1}{\Gamma(r)} \int_{1}^{t}\left(\log \frac{t}{s}\right)^{r-1} \nu(s) \frac{d s}{s}
$$

From (H1)-(H3), for each $t \in J$, we have

$$
\begin{aligned}
|h(t)| & \leq|y(1)|+\left|\frac{1}{\Gamma(r)} \int_{1}^{T}\left(\log \frac{T}{s}\right)^{r-1} \frac{\nu(s)}{s} d s\right| \\
& \leq \min \{|u(1)|,|w(1)|\}+\frac{1}{\Gamma(r)} \int_{1}^{T}\left(\log \frac{T}{s}\right)^{r-1} \frac{|\nu(s)|}{s} d s \\
& \leq \min \{|u(1)|,|w(1)|\}+\frac{\left\|\phi_{1}\right\|_{L_{1}}}{\Gamma(r+1)}(\log T)^{r} .
\end{aligned}
$$

Thus

$$
\|h\|_{\infty} \leq R .
$$

Step 3: $N$ maps bounded sets into equicontinuous sets of $B$. Let $t_{1}, t_{2} \in J, t_{1}<t_{2}$, Let $y \in B$ and $h \in N(y)$. Then 


$$
\begin{aligned}
\left|h\left(t_{2}\right)-h\left(t_{1}\right)\right| & =\mid \frac{1}{\Gamma(r)} \int_{1}^{t_{1}}\left[\left(\log \frac{t_{2}}{s}\right)^{r-1}-\left(\log \frac{t_{1}}{s}\right)^{r-1}\right] \nu(s) \frac{d s}{s} \\
& +\frac{1}{\Gamma(r)} \int_{t_{1}}^{t_{2}}\left(\log \frac{t_{2}}{s}\right)^{r-1} \nu(s) \frac{d s}{s} \mid \\
& \leq \frac{\left\|\phi_{1}\right\|_{L_{1}}}{\Gamma(r)} \int_{1}^{t_{1}}\left[\left(\log \frac{t_{2}}{s}\right)^{r-1}-\left(\log \frac{t_{2}}{s}\right)^{r-1}\right] \frac{d s}{s} \\
& +\frac{\left\|\phi_{1}\right\|_{L_{1}}}{\Gamma(r)} \int_{t_{1}}^{t_{2}}\left(\log \frac{t_{2}}{s}\right)^{r-1} \frac{d s}{s} \\
& \rightarrow 0 a s t_{1} \rightarrow t_{2} .
\end{aligned}
$$

As a consequence of the three above steps, we can conclude from the ArzeláAscoli theorem that $N: C(J, \mathbb{R}) \rightarrow \mathcal{P}(C(J, \mathbb{R}))$ is completely continuous.

Step 4: $N$ has a closed graph.

Let $y_{n} \rightarrow y_{*}, h_{n} \in N\left(y_{n}\right)$ and $h_{n} \rightarrow h_{*}$. We need to show that $h_{*} \in N\left(y_{*}\right)$. $h_{n} \in N\left(y_{n}\right)$ means that there exists $\nu_{n} \in \widetilde{S}_{F \circ \tau(y)}^{1}$ such that, for each $t \in J$,

$$
h_{n}(t)=y(1)+\frac{1}{\Gamma(r)} \int_{1}^{t}\left(\log \frac{t}{s}\right)^{r-1} \nu_{n}(s) \frac{d s}{s} .
$$

We must show that there exists $\nu_{*} \in \widetilde{S}_{F \circ \tau\left(y_{*}\right)}^{1}$ such that, for each $t \in J$,

$$
h_{*}(t)=y(1)+\frac{1}{\Gamma(r)} \int_{1}^{t}\left(\log \frac{t}{s}\right)^{r-1} \nu_{*}(s) \frac{d s}{s} .
$$

Since $F(t, \cdot)$ is upper semi-continuous, then for every $\epsilon>0$, there exists a natural number $n_{0}(\epsilon)$ such that, for every $n \geq n_{0}$, we have

$$
\nu_{n}(t) \in F\left(t, \tau y_{n}(t)\right) \subset F\left(t, y_{*}(t)\right)+\epsilon B(0,1) \quad \text { a.e. } t \in J .
$$

Since $F(\cdot, \cdot)$ has compact values, then there exists a subsequence $\nu_{n_{m}}(\cdot)$ such that

$$
\nu_{n_{m}}(\cdot) \rightarrow \nu_{*}(\cdot) \text { as } m \rightarrow \infty
$$

and

$$
\nu_{*}(t) \in F\left(t, \tau y_{*}(t)\right) \text { a.e. } t \in J .
$$

For every $w \in F\left(t, \tau y_{*}(t)\right)$, we have

$$
\left|\nu_{n_{m}}(t)-\nu_{*}(t)\right| \leq\left|\nu_{n_{m}}(t)-w\right|+\left|w-\nu_{*}(t)\right| .
$$

Then

$$
\left|\nu_{n_{m}}(t)-\nu_{*}(t)\right| \leq d\left(\nu_{n_{m}}(t), F\left(t, \tau y_{*}(t)\right) .\right.
$$


We obtain an analogous relation by interchanging the roles of $v_{n_{m}}$ and $v_{*}$, and it follows that

$$
\left|\nu_{n_{m}}(t)-\nu_{*}(t)\right| \leq H_{d}\left(F\left(t, \tau y_{n}(t)\right), F\left(t, \tau y_{*}(t)\right)\right) \leq l(t)\left\|y_{n}-y_{*}\right\|_{\infty} .
$$

Then

$$
\begin{aligned}
\left|h_{n_{m}}(t)-h_{*}(t)\right| & \leq \frac{1}{\Gamma(r)} \int_{1}^{t}\left(\log \frac{t}{s}\right)^{r-1}\left|\nu_{n_{m}}(s)-\nu_{*}(s)\right| \frac{d s}{s} \\
& \leq \frac{1}{\Gamma(r+1)}(\log T)^{r} \int_{1}^{T} l(s) d s\left\|y_{n_{m}}-y_{*}\right\|_{\infty} .
\end{aligned}
$$

Thus

as $m \rightarrow \infty$.

$$
\left\|h_{n_{m}}-h_{*}\right\|_{\infty} \leq \frac{1}{\Gamma(r+1)}(\log T)^{r} \int_{1}^{T} l(s) d s\left\|y_{n_{m}}-y_{*}\right\|_{\infty} \longrightarrow 0
$$

Hence, Lemma 2.1 implies that $N$ is upper semicontinuous.

Step 5: Every solution y of (3) - (4) satisfies

$$
u(t) \leq y(t) \leq w(t) \text { for all } t \in J .
$$

Let $y$ be a solution of $(3)-(4)$. We prove that

$$
u(t) \leq y(t) \text { for all } t \in J
$$

Suppose not. Then there exist $t_{1}, t_{2}$ with $t_{1}<t_{2}$ such that $u\left(t_{1}\right)=y\left(t_{1}\right)$ and

$$
u(t)>y(t) \text { for all } t \in\left(t_{1}, t_{2}\right) .
$$

In view of the definition of $\tau$ one has

$$
{ }^{H c} D^{r} y(t) \in F(t, u(t)) \text { for all } t \in\left(t_{1}, t_{2}\right) .
$$

Thus there exists $v \in S_{F \circ \tau(u)}$ with $v(t) \geq v_{1}(t)$ a.e. on $\left(t_{1}, t_{2}\right)$ such that

$$
{ }^{H c} D^{r} y(t)=v(t) \text { for all } t \in\left(t_{1}, t_{2}\right) .
$$

An integration on $\left(t_{1}, t\right]$, with $t \in\left(t_{1}, t_{2}\right)$ yields

$$
y(t)-y\left(t_{1}\right)=\frac{1}{\Gamma(r)} \int_{t_{1}}^{t}\left(\log \frac{t}{s}\right)^{r-1} \nu(s) \frac{d s}{s} .
$$

Since $u$ is a lower solution to $(1)-(2)$, then

$$
u(t)-u\left(t_{1}\right) \leq \frac{1}{\Gamma(r)} \int_{t_{1}}^{t}\left(\log \frac{t}{s}\right)^{r-1} v_{1}(s) \frac{d s}{s} ; t \in\left(t_{1}, t_{2}\right) .
$$

It follows from $y\left(t_{1}\right)=u\left(t_{1}\right)$ and $\nu(t) \geq v_{1}(t)$ that

$$
u(t) \leq y(t) \text { for all } t \in\left(t_{1}, t_{2}\right) .
$$

This is a contradiction, since $u(t)>y(t)$ for all $t \in\left(t_{1}, t_{2}\right)$. Consequently

$$
u(t) \leq y(t) \text { for all } t \in J .
$$


Analogously, we can prove that

$$
y(t) \leq w(t) \text { for all } t \in J .
$$

This shows that

$$
u(t) \leq y(t) \leq w(t) \text { for all } t \in J .
$$

Consequently, the problem (3) - (4) has a solution $y$ satisfying $u \leq y \leq w$.

Step 6: Every solution of problem (3) - (4) is solution of (1) - (2). Suppose that $y$ is a solution of problem (3) - (4). Then, we have

$$
{ }^{H c} D^{r} y(t) \in F(t, \tau(y(t))) \text { for a.e. } t \in J,
$$

and

$$
y(1)=\tau(y(1))-L(\bar{y}(1), \bar{y}(T)) .
$$

Since, for all $t \in J$, we have

$$
u(t) \leq y(t) \leq w(t)
$$

then,

$$
\tau(y(t))=y(t) .
$$

Thus, we get

$$
{ }^{H c} D^{r} y(t) \in F(t, y(t)) \text { for a.e. } t \in J,
$$

and

$$
L(y(1), y(T))=0 .
$$

We only need to prove that

$$
u(1) \leq y(1)-L(y(1), y(T)) \leq w(1) .
$$

Suppose that

$$
y(1)-L(y(1), y(T))<u(1) .
$$

Since $L(u(1), u(T)) \leq 0$, we have

$$
y(1) \leq y(1)-L(u(1), u(T)) .
$$

Since $L(\cdot, \cdot)$ is nonincreasing with respect to its both arguments, then we obtain

$$
y(1) \leq y(1)-L(u(1), u(T)) \leq y(1)-L(y(1), y(T))<u(1) .
$$

Hence, we get $y(1)<u(1)$, which is a contradiction. Analogously we can prove that

$$
y(1)-L(y(1), y(T)) \leq w(1) .
$$

Hence, $y$ is a solution to $(1)-(2)$.

This concludes that problem (1) - (2) has a solution $y$ satisfying $u \leq y \leq$ $w$. 
Remark 3.2. In the case where $L(x, y)=a x-b y-c$, Theorem 3.1 shows existence results to the following problem,

$$
\begin{gathered}
{ }^{H c} D^{r} y(t) \in F(t, y(t)) \text { for a.e. } t \in J, \\
a y(1)-b y(T)=c,
\end{gathered}
$$

where $-b<a \leq 0 \leq b, c \in \mathbb{R}$, which includes the anti-periodic case $b=$ $-a, c=0$.

\section{REFERENCES}

[1] S. Abbas, M. Benchohra, Upper and lower solutions method for Darboux problem for fractional order implicit impulsive partial hyperbolic differential equations, Acta Univ. Palacki. Olomuc., 51 (2) (2012), pp. 5-18.

[2] S. Abbas, M. Benchohra, The method of upper and lower solutions for partial hyperbolic fractional order differential inclusions with impulses, Discuss. Math. Differ. Incl. Control Optim., 30 (1) (2010), pp. 141-161.

[3] S. Abbas, M. Benchohra, M. Bohner, Weak solutions for implicit differential equations of Hilfer-Hadamard fractional derivative, Adv. Dyn. Syst. Appl., 12 (1) (2017), pp. $1-16$.

[4] S. Abbas, M. Benchohra, J. R. Graef, J. Henderson, Implicit Fractional Differential and Integral Equations: Existence and Stability, De Gruyter, Berlin, 2018.

[5] S. Abbas, M. Benchohra, A. Hammoudi, Upper, lower solutions method and extremal solutions for impulsive discontinuous partial fractional differential inclusions, Panamer. Math. J., 24 (1) (2014), pp. 31-52.

[6] S. Abbas, M. Benchohra, J. E. Lazreg, Y. Zhou, A Survey on Hadamard and Hilfer fractional differential equations: Analysis and Stability, Chaos, Solitons Fractals, 102 (2017), pp. 47-71.

[7] S. Abbas, M. Benchohra, G. M. N'Guérékata, Topics in Fractional Differential Equations, Springer, New York, 2012.

[8] S. Abbas, M. Benchohra, G. M. N'Guérékata, Advanced Fractional Differential and Integral Equations, Nova Science Publishers, New York, 2015.

[9] S. Abbas, M. Benchohra, J. J. Trujillo, Upper and lower solutions method for partial fractional differential inclusions with not instantaneous impulses, Prog. Frac. Diff. Appl., 1 (1) (2015), pp. 11-22.

[10] Y. Adjabi, F. Jarad, D. Baleanu, T. Abdeljawad, On Cauchy problems with Caputo Hadamard fractional derivatives, J. Comput. Anal. Appl., 21 (4) (2016), pp. 661-681.

[11] P. Agarwal, Some inequalities involving Hadamard-type $k$-fractional integral operators, Math. Methods Appl. Sci., 40 (11) (2017), pp. 3882-3891.

[12] P. Agarwal, J. J. Nieto, M. -J. Luo, Extended Riemann-Liouville type fractional derivative operator with applications, Open Math., 15 (1) (2017), pp. 1667-1681. 
[13] B. Ahmad, S. K. Ntouyas, Initial value problems for hybrid Hadamard fractional equations, Electron. J. Differential Equations, 161 (2014), pp. 1-8.

[14] M. Benchohra, S. Hamani, The method of upper and lower solution and impulsive fractional differntial inclusions, Nonlinear Anal: Hybrid Syst., 3 (2009), pp. 433-440.

[15] M. Benchohra, S. K. Ntouyas, The lower and upper method for first order differential inclusions with nonlinear boundary condition, J. Inequ. Pure Appl. Math., 3 (1) (2002), Article 14, pp. 1-20.

[16] H. F. Bohnenblust, S. Karlin, On a theorem of Ville. Contribution on the theory of games, in: Annals of Mathematics Studies, Vol. 24, Princeton University Press, Princeton, NJ, 1950, pp. 155-160.

[17] R. Hilfer, Applications of Fractional Calculus in Physics, World Scientific, Singapore, 2000 .

[18] Sh. Hu, N. Papageorgiou, Handbook of Multivalued Analysis, Volume I: Theory, Kluwer, Dordrecht, Boston, London, 1997.

[19] F. Jarad, T. Abdeljawad, D. Baleanu, Caputo-type modification of the Hadamard fractional derivatives, Adv. Diff. Equa., 142 (2012), pp. 1-12.

[20] A. A. Kilbas, H. M. Srivastava, J. J. Trujillo, Theory and Applications of Fractional Differential Equations, North-Holland Mathematics Studies, Vol. 204, Elsevier Science B.V., Amsterdam, 2006.

[21] I. O. Kiymaz, P. Agarwal, S. Jain, A. Cetinkaya, On a new extension of Caputo fractional derivative operator, in: Advances in Real and Complex Analysis with Applications, pp. 261-275, Trends Math., Birkhauser/Springer, Singapore, 2017.

[22] G. S. Ladde, V. Lakshmikantham, A. S. Vatsala, Monotone Iterative Technique for Nonlinear Differential Equations, Pitman Advanced Publishing Program, London, 1985.

[23] V. F. Morales-Delgado, J. F. Gomez-Aguilar, K. M. Saad, M. A. Khan, P. Agarwal, Analytic solution for oxygen diffusion from capillary to tissues involving external force effects: a fractional calculus approach, Phys. A, 523 (2019), pp. 48-65.

[24] I. Podlubny, Fractional Differential Equations, Academic Press, San Diego, 1999.

[25] G. Singh, P. Agarwal, M. Chand, S. Jain, Certain fractional kinetic equations involving generalized $k$-Bessel function, Trans. A. Razmadze Math. Inst., 172 (3), part B (2018), pp. 559-570. 


\section{SAÏD AbBas}

Laboratory of Mathematics, Geometry,

Analysis, Control and Applications

Tahar Moulay University of SaÏDA

P.O. Box 138, EN-NAsR, 20000 SAÏDA

Algeria

E-mail address: abbasmsaid@yahoo.fr

\section{MoufFak Benchohra}

LABORATORY OF MATHEMATiCS

Duillali Liabes University of Sidi Bel-Abbès

P.O. Box 89, Sidi Bel-Abbès 22000

Algeria

E-mail address: benchohra@univ-sba.dz

\section{SAmira Hamani}

aboratoire des Mathematiques Appliqués et Pures Université de Mostaganem

B.P. 227, 27000, Mostaganem

Algeria

E-mail address: hamani_samira@yahoo.fr

\section{JOHNNY HENDERSON}

Department of Mathematics

BAYLOR UNIVERSITY

WaCO, TEXAS 76798-7328

USA

E-mail address: johnny_henderson@baylor.edu 